\title{
Optical waveguide cantilever actuated by light
}

\author{
Kirill Zinoviev, ${ }^{\text {a) }}$ Carlos Dominguez, Jose Antonio Plaza, and Laura M. Lechuga \\ National Centre for Microelectronics (IMB-CSIC), Campus UAB, Cerdanyola del Valles, 08193 Barcelona, \\ Spain
}

(Received 27 September 2007; accepted 7 December 2007; published online 4 January 2008)

\begin{abstract}
We present experimental studies and theoretical analysis of a microcantilever working as an optical waveguide metallized from one side and actuated by light propagating inside it. Light absorbed in the metallic layer increases the temperature of the bimetallic structure and induces the cantilever bending. Dynamical properties of the cantilevers were studied using actuation by light modulated at frequencies of up to $800 \mathrm{~Hz}$. The deflection of the cantilever by $400 \mathrm{~nm}$ was obtained while $0.25 \mathrm{~mW}$ of light power was coupled inside it. (C) 2008 American Institute of Physics.
\end{abstract}

[DOI: $10.1063 / 1.2830818$ ]

Over the past decades, the microcantilevers have demonstrated dramatic impact on the development of biological, physical, and chemical sensors. Further improvements can be achieved by scaling down their sizes, increasing the integration level, and improving the readout techniques. The cantilevers are used in both static deflection and resonance modes $^{1,2}$ where the initial displacement and excitation of them are the special issues which should be properly addressed when designing the experiments on their basis. In static mode, the cantilevers in arrays frequently suffer chaos in the initial displacement of them. Special cantilever geometry, postfabrication processing, or experimental setup modification are frequently required for correction of the initial offset. ${ }^{3,4}$ The latter can be also controlled by means of heating the cantilever with a laser. The photothermal actuation is attractive, especially when the cantilever is operating in the resonant mode ${ }^{5-8}$ Normally, light from a laser diode with an output power of a few milliwatts is focused on microcantilever composed of at least two different materials. The cantilever can be deflected in one direction or it can be vibrated if the modulated light is applied. The cantilever deflection depends much on the geometry and the location of the laser spot.

In this work, we studied the optical and mechanical properties of gold coated optical waveguide cantilevers (OWCs). The cantilevers were actuated by light propagating inside them. This approach offers an alternative to the known methods for microactuating and allows for further integration of the devices containing microcantilevers on chip. The cantilever deflection was monitored using the optical beam deflection technique.

Schematic view and the scanning electron microscopy (SEM) image of OWC used in the experiment are shown in Fig. 1. The device was fabricated using the technology reported elsewhere. ${ }^{9}$ The waveguide microcantilever made of silicon dioxide is an extension of the silicon dioxide layer thermally grown on silicon substrate. The developed technology allowed fabrication of stress-free silicon dioxide cantilevers. This is achieved by eliminating the bottom layer of the cantilevers after they are released from the substrate. Light is coupled into the cantilever from a silicon nitride input waveguide (IW) which is terminated right where the cantilever

a) Author to whom correspondence should be addressed. Electronic mail: kirill.zinoviev@cnm.es. Tel.: +349 35947700. FAX: +345 801496. begins. The light propagates until the end of the cantilever and couples into the output waveguide separated from the cantilever by a short gap of $3 \mu \mathrm{m}$. The coupling efficiency to the output waveguide is a function of the cantilever deflection. ${ }^{9}$

The cantilever beam is $600 \mathrm{~nm}$ thick, $200 \mu \mathrm{m}$ long, and $40 \mu \mathrm{m}$ wide; the cantilever fundamental resonance mode can be excited at frequency of about $13 \mathrm{kHz} .{ }^{9}$ The cantilever waveguide supports two propagating modes for the light wavelength of $633 \mathrm{~nm}$. A gold layer with thickness of $\sim 10 \mathrm{~nm}$ was deposited on the bottom surface of the cantilever using the electron beam sputtering. No intermediate layer was applied to improve the adhesion of the metal to silicon dioxide.

Intensity of light propagating along the cantilever is decreasing according to the expression

$$
P(x)=P_{0} \exp (-\alpha x),
$$

where $\alpha$ is an absorption coefficient which is a function of the cantilever parameters and of the mode order. $P_{0}$ is the power supplied to the cantilever (at $x=0$ ). Absorption coefficient presented on graph in Fig. 2 as a function of the gold layer thickness was calculated for two modes and two differ-

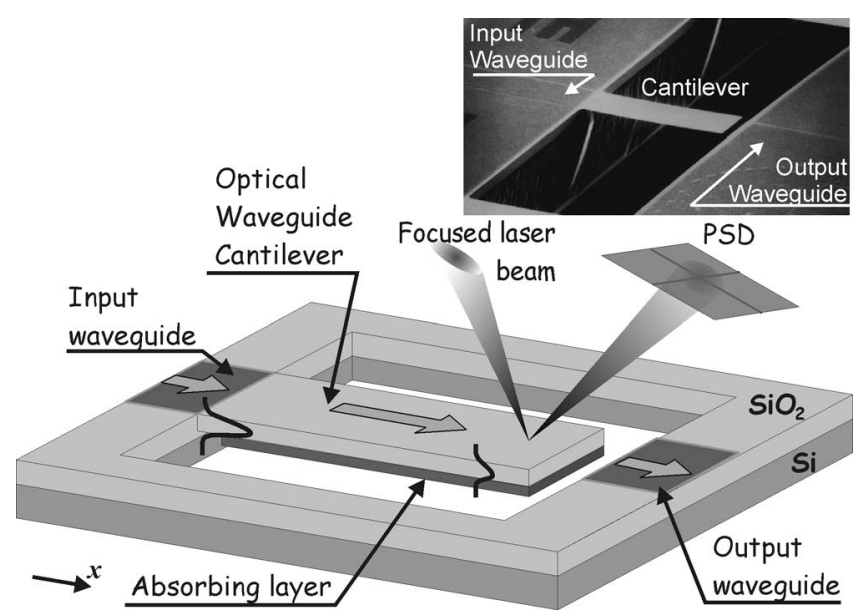

FIG. 1. A schematic view of a cantilever with an absorption gold layer. The decaying fundamental mode propagating along the waveguide is shown. Cantilever is optically coupled with the silicon nitride input waveguide used for light delivery. The output waveguide butt coupled with the OWC is separated from the cantilever free end by a short gap of $3 \mu \mathrm{m}$. In the inset, SEM image of the cantilever is presented. 


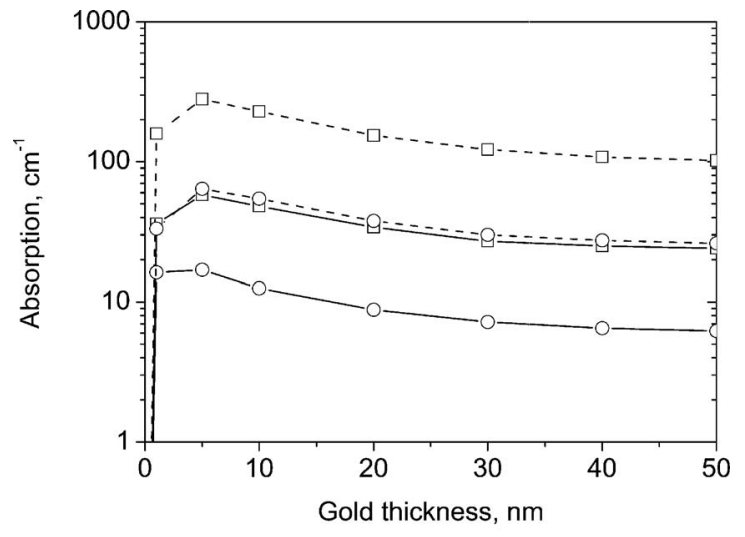

FIG. 2. Absorption coefficient of a silicon oxide waveguide cantilever with gold layer on one side versus the thickness of gold layer. Squares and circles stand for $600 \mathrm{~nm}$ and $1000 \mathrm{~nm}$ thick waveguides, respectively. Absorption for the fundamental and for the first order modes is denoted by solid and dash lines, respectively.

ent cantilever thicknesses. The simulations for $1000 \mathrm{~nm}$ thick cantilevers were performed to see how strong the influence of the thickness on the absorption losses is. As it was expected, the absorption is higher for the first order mode and thin cantilevers. Theoretically, $38 \%$ of light would reach the end of a $600 \mathrm{~nm}$ thick, $200 \mu \mathrm{m}$ long waveguide cantilever, covered with $10 \mathrm{~nm}$ of gold, if working with the fundamental mode, and only $1 \%$ in the case of the first order mode. All the calculations were performed using the transfer matrix approach. ${ }^{10}$ The wavelength of $633 \mathrm{~nm}$ and TE polarization were considered for the simulations.

The power density in gold layer along the cantilever can be described by the expression

$$
D_{a}(x)=\frac{P_{0} \alpha \exp (-\alpha x)}{w t},
$$

where $w$ and $t$ are the width and the thickness of the gold layer, respectively. According to the simulations, the energy density absorbed and converted in heat decreases toward the cantilever end by approximately a factor of 2 , almost regardless the gold layer thickness. The energy density is around $5 \times 10^{12} \mathrm{~W} / \mathrm{m}^{3}$ if $1 \mathrm{~mW}$ of energy is coupled to the cantilever.

The stationary problem of temperature distribution along the cantilever was solved using the commercially available software (ANSYS). The model considered in the simulation had the parameters close to the ones of the device used in the experiment. A gold layer of $10 \mathrm{~nm}$ was assumed to be deposited onto the bottom of $600 \mathrm{~nm}$ thick, $40 \mu \mathrm{m}$ wide, and $200 \mu \mathrm{m}$ long silica cantilever suspended from a silicon substrate in air. It was assumed that $0.05 \mathrm{~mW}$ of $\mathrm{cw}$ light irradiation was coupled into the beam. The results of the simulation showed that the temperature (relative to the ambient temperature) is almost $5^{\circ}$ in the hottest point. It gradually decreases to reach $2.2^{\circ}$ at the apex and drops down to $0^{\circ}$ at the anchoring area.

Simple models are available for thermally induced stresses in microcantilevers consisting of two layers of materials with different coefficients of thermal expansion. ${ }^{1}$ The deformation, referred to as a bimetallic effect, makes the cantilever to bend forming a profile with a certain radius of curvature along the cantilever so that the displacement of the cantilever free end may be expressed as ${ }^{3}$

$$
\Delta z=\frac{3 l^{2}\left(\alpha_{1}-\alpha_{2}\right) \Delta T}{t_{1}+t_{2}}\left[\frac{\left(1+\frac{t_{1}}{t_{2}}\right)^{2}}{3\left(1+\frac{t_{1}}{t_{2}}\right)^{2}+\left(1+\frac{t_{1} E_{1}}{t_{2} E_{2}}\right)\left(\frac{t_{1}^{2}}{t_{2}^{2}}+\frac{t_{2} E_{2}}{t_{1} E_{1}}\right)}\right]
$$

where $t_{1}$ and $t_{2}$ are the thicknesses of the two layers of the bimaterial structure, $E_{1}$ and $E_{2}$ are the Young's moduli, and $\alpha_{1}$ and $\alpha_{2}$ are the thermal expansion coefficients of these layers, respectively. Calculations made using this formula gave deflection of $80 \mathrm{~nm}$ per degree of temperature change. Thus, the cantilever, if uniformly heated by $5^{\circ}$, will be deflected by $400 \mathrm{~nm}$. The assumption of uniformly distributed heat along the cantilever was made while using this formula. However, in our experiment, the temperature distribution is nonuniform, so the cantilever deflection will be less than the magnitude predicted by formula (3).

In order to estimate the time constant of the cantilever, the orthogonal expansion technique ${ }^{11}$ for a multilayer medium with perfect contact between layers was adapted to the two-dimensional transient model describing the metallized cantilever. The problem solution involves the solution of steady state problem followed by the solution of the transient one. The solution strongly depends, among the other factors,

Downloaded 28 Jan 2008 to 158.109 .6 .83 . Redistribution subjec on the average heat transfer coefficients describing the thermal exchange between the cantilever and the surrounding media. The time constant of the order of $1 \mathrm{~ms}$ was obtained when the coefficient was taken of the order of $2 \times 10^{3}$.

In the experiment, a light beam from a $\mathrm{HeNe}$ laser $(632.8 \mathrm{~nm}, 10 \mathrm{~mW})$ was focused by an objective lens to the facet of the input waveguide. Light power coupled to the cantilever was measured before deposition of gold layer. The coupled power was about $0.25 \mathrm{~mW}$. It was assumed that by introducing the gold layer, we did not affect much the coupling efficiency. Modulation of light intensity was realized with a mechanical chopper. Light beam from another $\mathrm{HeNe}$ laser $(632.8 \mathrm{~nm}, 1 \mathrm{~mW})$ was focused by a planoconvex lens at the apex of the cantilever and reflected to a position sensitive photodetector (optical beam deflection technique).

Because of the nonsymmetrical architecture of the junction IW-OWC, the light was coupled both into the fundamental and the first order modes. Moving the cantilever up 


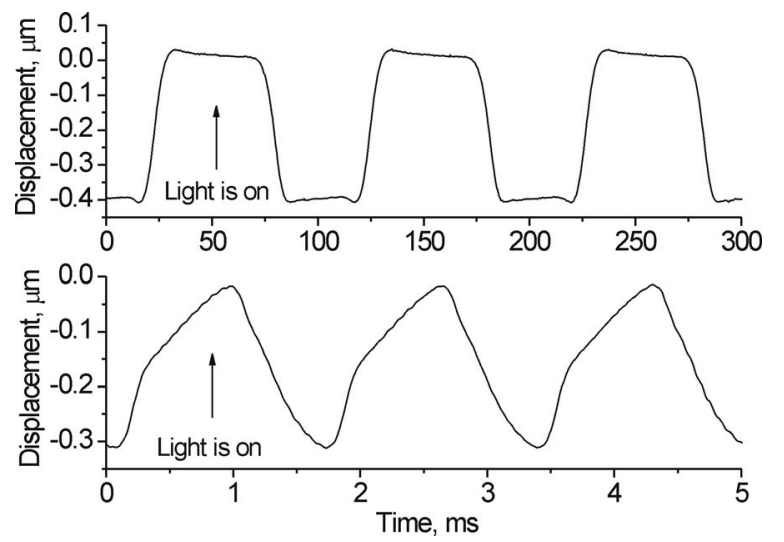

FIG. 3. Time diagram of the cantilever deflection induced by light coupled inside the cantilever and modulated with frequency of (a) $100 \mathrm{~Hz}$ and (b) $600 \mathrm{~Hz}$.

and down by air flux and monitoring the power of light coupled into the output waveguide, we scanned the distribution of light intensity at the cantilever exit. The close to Gaussian distribution of the intensity indicated that fundamental mode mostly reached the exit.

Light was modulated with frequencies lying in a range of $0-800 \mathrm{~Hz}$. The amplitude of cantilever vibration is a function of frequency due to thermal inertia of the structure. The amplitude gradually decreases starting from $400 \mathrm{~Hz}$. When at $800 \mathrm{~Hz}$, the amplitude drops down to a half of that measured at $100 \mathrm{~Hz}$. The time diagram of the cantilever motion induced by external light modulation is presented in Fig. 3 . The modulation with chopper does not allow monitoring the dynamics of the cantilever behavior at low frequencies where the cantilever motion is mainly defined by the shape of the light pulses [see Fig. 3(a)]. The diameter of the laser beam was comparable with the slit width in the chopper blade and the rise time of the pulses depended on the modulation frequency. The light pulses had a rise time of near $30 \%$ of pulse duration. Therefore, the cantilever motion is a function of light pulse shape and cantilever deflection response time. At higher frequencies, when the rise time of the light pulse is comparable with the cantilever response time, the cantilever dynamics starts to depend on inertia of heat propagation.

This can be observed in Fig. 3(b) where the cantilever free end trajectory is presented; light was modulated with a frequency of $600 \mathrm{~Hz}$. Due to thermal inertia, the beam did not have enough time to get to initial position which is around $-400 \mathrm{~nm}$ and did not reach the maximum deflection point, potentially reachable for this power at low frequencies. The trajectory is "distorted;" it has a steep rise until half of the amplitude, and then relatively slow increment until the maximum. This is due to the influence of both finite light pulse rise time and finite cantilever response time. Flattop of the curves on the diagrams disappeared at approximately $400 \mathrm{~Hz}$. This is how the response time of the metallized cantilever was determined to be $1.25 \mathrm{~ms}$. The response time of the same order of magnitude on the cantilevers with similar geometry was reported in Ref. 5.

The presented bimetallic structure is a device with the response time of about $1.25 \mathrm{~ms}$ determined experimentally. According to the simulations, with $0.25 \mathrm{~mW}$ of energy supplied inside it, the cantilever was heated to a temperature of a few degrees. The heating with this power results in the cantilever deflection by more than $400 \mathrm{~nm}$ nanometers at frequencies lower than $100 \mathrm{~Hz}$. At higher frequencies the amplitude of vibration goes down and one cannot expect high deflection amplitudes if the cantilever is actuated in the frequency range corresponding to the fundamental resonance mode, which is near $13 \mathrm{kHz}$ for the presented device. It was experimentally observed that the fundamental waveguide mode is able to propagate until the end of a $200 \mu \mathrm{m}$ long metallized cantilever with the losses which allow light coupling into the output waveguide and the detection of it at the exit of this waveguide.

This work was supported by the Spanish National Research Council (CSIC), project Optobiocel, and by M. Boutin Foundation. K. Zinoviev acknowledges the financial support from I3P program (Spain).

${ }^{1}$ N. V. Lavrik, M. J. Sepaniak, and P. G. Datskos, Rev. Sci. Instrum. 75, 2229 (2004)

${ }^{2}$ L. G. Carrascosa, M. Moreno, M. Alvarez, and L. M. Lechuga, Trends Analyt. Chem. 25, 196 (2006).

${ }^{3}$ J. A. Plaza, K. Zinoviev, G. Villanueva, M. Álvarez, J. Tamayo, C. Domínguez, and L. M. Lechuga, Appl. Phys. Lett. 89, 094109 (2006).

${ }^{4}$ X. R. Zhang and X. F. Xu, Appl. Phys. Lett. 86, 021114 (2005).

${ }^{5}$ J. K. Gimzewski, Ch. Gerber, E. Meyer, and R. R. Schlittler, Chem. Phys. Lett. 217, 589 (1994).

${ }^{6}$ N. V. Lavrik and P. G. Datskos, Appl. Phys. Lett. 82, 2697 (2003).

${ }^{7}$ M. Moulin, R. J. Stephenson, and M. E. Welland, J. Vac. Sci. Technol. B 15, 590 (1997).

${ }^{8}$ L.-H. Han and S. Chen, Sens. Actuators, A 121, 35 (2005).

${ }^{9}$ K. E. Zinoviev, C. Dominguez, J. A. Plaza, V. Cadarso, and L. M. Lechuga, J. Lightwave Technol. 24, 2132 (2006).

${ }^{10}$ E. Anemogeannis and E. N. Glytsis, J. Lightwave Technol. 10, 1344 (1992).

${ }^{11}$ M. N. Özisik, Heat Conduction, 2nd ed. (Wiley, New York, 1993). 